\title{
RELIABILITY EVALUATION OF THICK FILM RESISTORS THROUGH MEASUREMENT OF THIRD HARMONIC INDEX
}

\author{
SUSUMU KASUKABE and MINORU TANAKA \\ Production Engineering Research Laboratory, Hitachi Ltd., Totsuka-ku, Yokohama 244
}

\begin{abstract}
The degradation mechanism of ruthenium-based thick film resistors is investigated in accelerated tests under various conditions of humidity, temperature and overload stress. This study shows that the variation of resistance is mainly caused by hydration and dehydration in the conductive component $\mathrm{RuO}_{2}: \mathrm{RuO}_{2}+\mathrm{xH}_{2} \mathrm{O} \leftrightarrow \mathrm{RuO}_{2} \cdot \mathrm{xH}_{2} \mathrm{O}$. In addition to measuring resistance, the Third Harmonic Index (THI) is studied as the characteristic which may indicate the degree of various defects in resistors. A strong correlation exists between "the initial THI" and "the variation rate of resistance during an accelerated test". A resistor which indicates a quite large initial THI shows remarkable variation of resistance, and has scratches and/or foreign substances on the surface of the thick film resistor. As a result, it becomes evident that the initial THI can be used to predict the stability of a resistor in a nondestructive test.
\end{abstract}

\section{INTRODUCTION}

Thick film resistors are widely used in microelectronic devices. Ruthenium-based resistor systems have especially high reliability. As a fast, simple and nondestructive measuring method, the Third Harmonic Index (THI) of a passive component has been studied by several researchers. ${ }^{1-3}$ These experiments have shown that the components which exhibit a relatively high nonlinearity are less stable. In this study we applied the measurement of THI to thick film resistors made especially for this experiment, and investigated the possibility of estimating reliability through measurement of THI. The results of a study of the degradation mechanism of a ruthenium-based thick film resistor, and of the relationship between the THI and the variation rate of resistance under various accelerated tests are presented.

\section{EXPERIMENTAL PROCEDURE}

\subsection{Specimen}

The resistor used for this investigation is shown schematically in Figure 1. Six thick film resistors of $56 \Omega$ are printed on an alumina substrate with 8-Lead, and this module resistor permits the use of each $56 \Omega$ element independently. Thick film resistors consist of ruthenium oxide, bismuth ruthenate and silicate glass.
Electrodes and common-conductors consist of $\mathrm{Ag}-\mathrm{Pd}$ and $\mathrm{Au}-\mathrm{Pt}$ based thick film conductors, respectively. The surface of the resistor is coated first with glass and then Si-based resin.

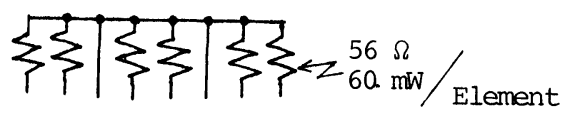

Resistor circuits

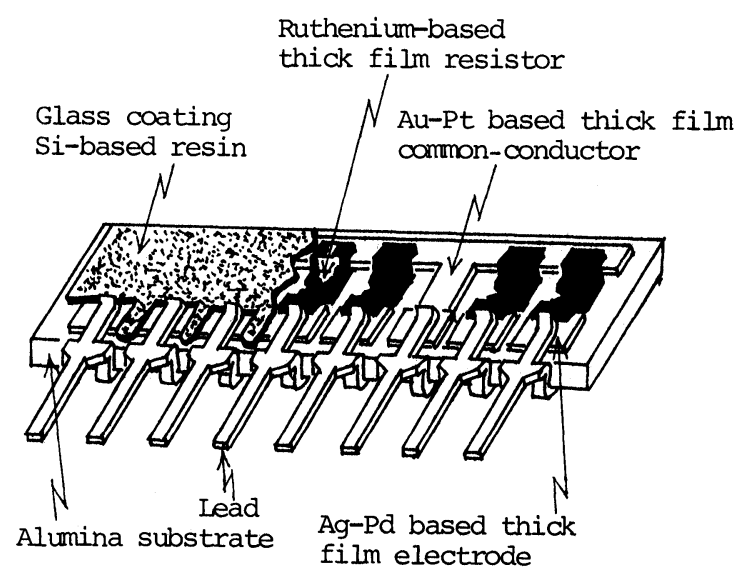

FIGURE 1 Schematic view of a thick film resistor. 


\subsection{Experimental Conditions}

The stability of ruthenium-based thick film resistors was investigated in accelerated tests under various conditions of humidity, temperature and overload stress as shown in Table I. Table II shows the measuring parameters and their methods. In addition to measuring resistance, the Third Harmonic Index (THI) is studied as the characteristic which may indicate the degree of various defects in resistors. ${ }^{2}$

TABLE I

Accelerated test conditions.

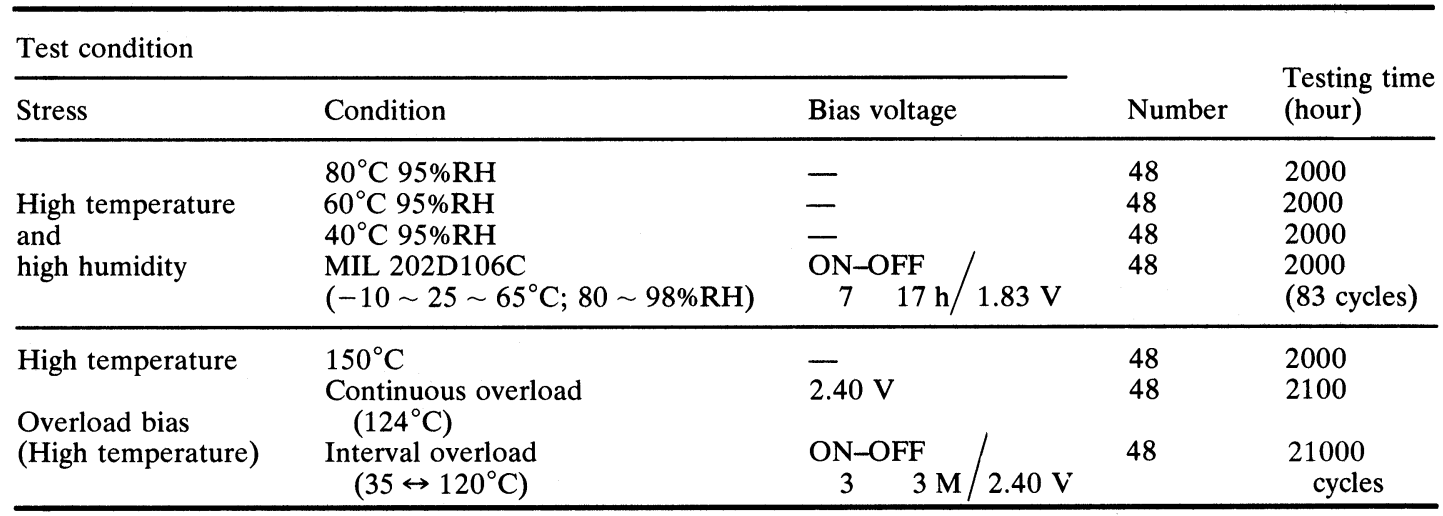

TABLE II

Measuring parameters and method.

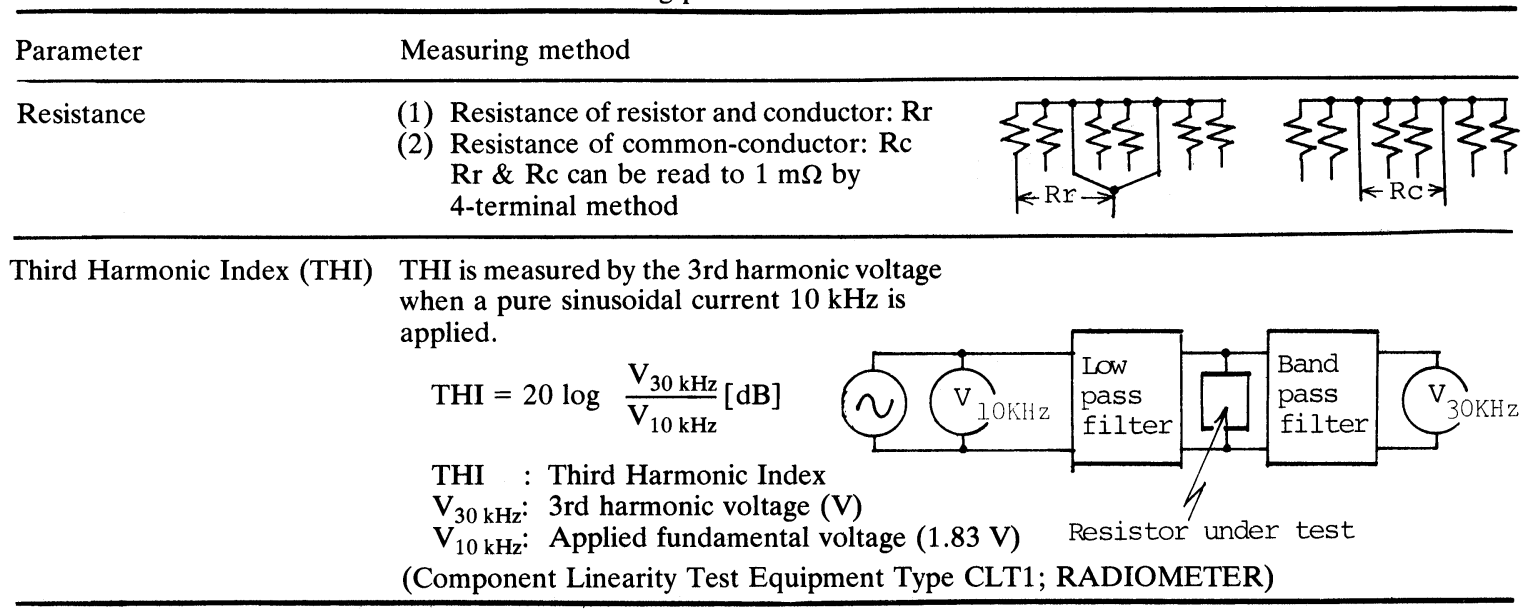

\section{EXPERIMENTAL RESULTS}

Experimental results indicated that there were two variation types of resistance: One was quite large, while the other was small. Therefore, the experimental results and discussion will be described in this paper by dividing these variation types into two groups.

\subsection{Distribution of the Initial THI}

The measurement principle of $\mathrm{THI}^{3}$ is shown schematically in Table II. In this investigation, a $10 \mathrm{kHz}$ pure sinusoidal current was fed through a low-pass filter to a $56 \Omega$ resistor under test. The $30 \mathrm{kHz}$ distortion generated in the resistor was amplified by the narrow-band $30 \mathrm{kHz}$ amplifier. THI, the degree of 
nonlinearity, is defined as the ratio between the 3rd harmonic voltage $\left(\mathrm{V}_{30 \mathrm{kHz}}\right)$ and the applied fundamental voltage $\left(\mathrm{V}_{10 \mathrm{kHz}}: 1.83 \mathrm{~V}\right)$ as shown in the following equation, expressed in $\mathrm{dB}$.

$$
\mathrm{THI}=20 \log \frac{V_{30 \mathrm{kHz}}}{V_{10 \mathrm{kHz}}}(\mathrm{dB})
$$

The measuring result on THI is shown in Figure 2. Figure 2 shows that this distribution consists of the bimodal lognormal distribution. The difference between the two groups becomes evident, as seen in the following paragraph.

For each of these specimens, an accelerated test as shown in Table I was carried out under various conditions of humidity, temperature and overload stress.

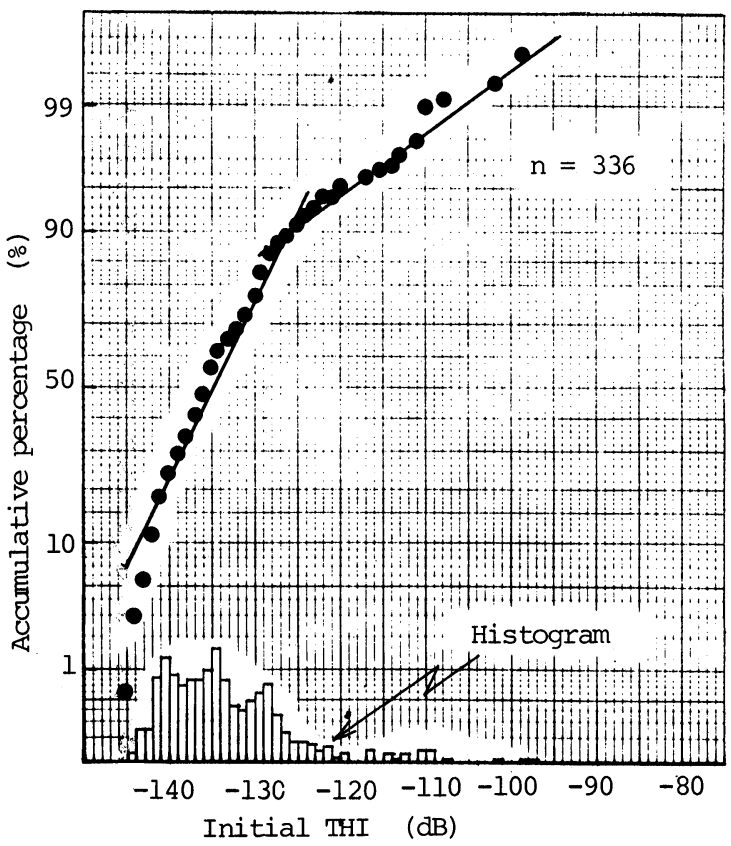

FIGURE 2 Distribution of initial Third Harmonic Indexes on a normal distribution paper.

\subsection{Results of Accelerated Test}

Through the self-heating of a resistor in the overload test, the continuous overloaded bias test was regarded as the high temperature storage test $\left(124^{\circ} \mathrm{C}\right)$; and similarly the interval overload bias test was regarded as the temperature cycling test $\left(35 \sim 120^{\circ} \mathrm{C}\right)$.

In this paper, the experimental results of these accelerated tests are considered as two groups as follows:

1) the high temperature storage test at $150^{\circ} \mathrm{C}$ and the overload tests put together into "a group under temperature stress", and

2) the high temperature and humidity storage tests, i.e. $80^{\circ} \mathrm{C} 95 \% \mathrm{RH}, 60^{\circ} \mathrm{C} 95 \% \mathrm{RH}$, and $40^{\circ} \mathrm{C} 95 \% \mathrm{RH}$, and the moisture resistance test (MIL STD 202D106C) put together into "a group under humidity stress".

\subsubsection{Large variation of resistance Figure 3 shows} the relationship between the initial THI and the variation rate of resistance (DR/Ro) in accelerated

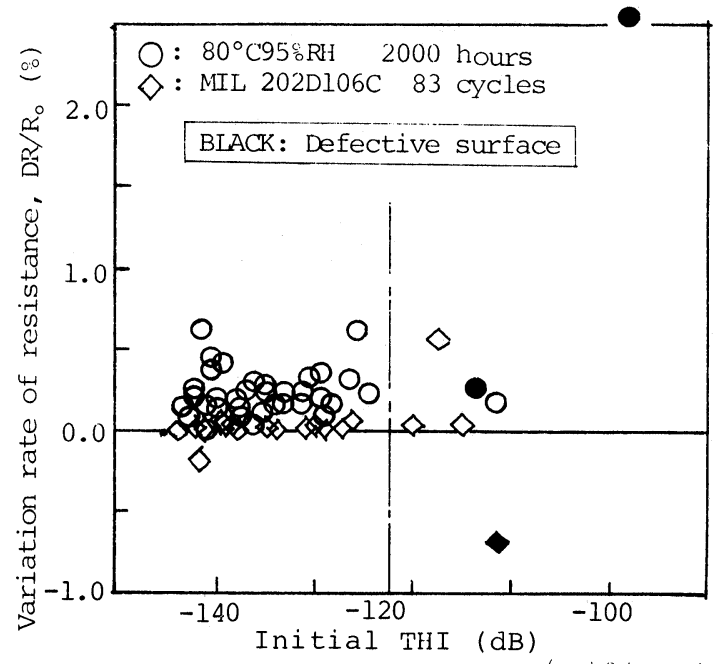

(a) High humidity stress

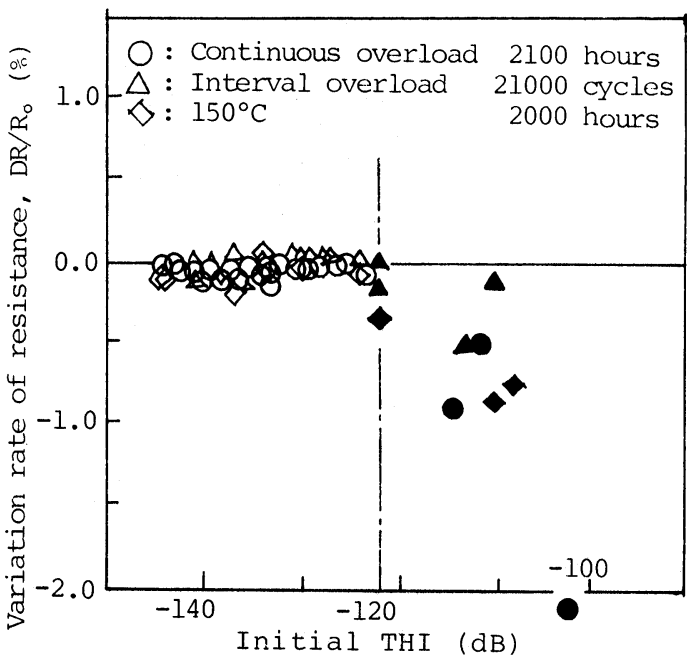

(b) High temperature stress $\left(n=4+\left(3 / T^{\prime}(2: i t)\right.\right.$

FIGURE 3 Relationship between initial Third Harmonic Index and variation rate of resistance. 

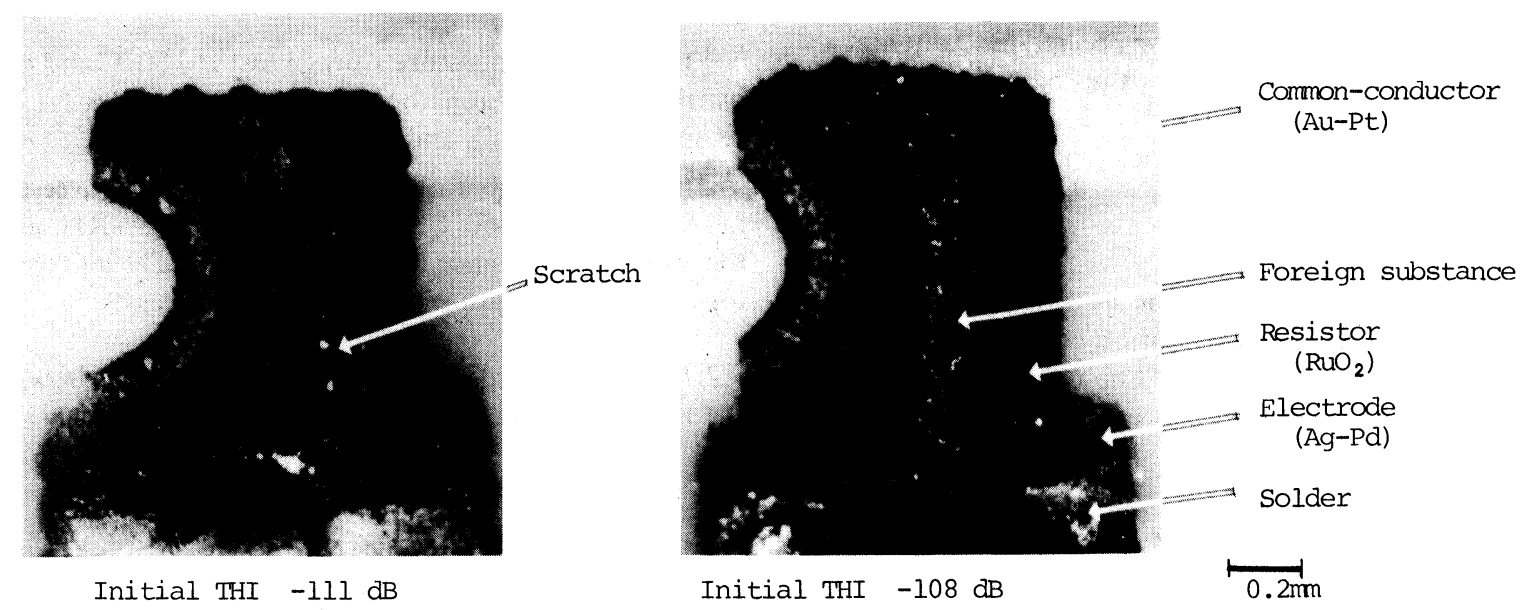

FIGURE 4 Scratches and foreign substances on the surface of thick film resistors.

tests after 2000 hours. This figure indicates that the specimens with large initial THI have a tendency to show a large variation rate of resistance. Surfaces of resistors were investigated after removing the Si-based resin. It was found that most of the resistors which showed remarkable resistance change had scratches and/or foreign substances on the surface of thick film resistors as shown in Figure 4. Energy-dispersive X-ray analysis revealed that these foreign substances consisted of $\mathrm{Cr}, \mathrm{Fe}$ and $\mathrm{Ni}$. It is considered that a defective surface was caused by a poor glass-coating process; e.g., the resistor touched the wire netting in the electric furnace. All specimens having a defective surface showed more than or equal to $-122 \mathrm{~dB}$ of THI, and they are marked in black in Fig. 3. These results indicate that the remarkable resistance change was mainly caused by the defective surface of a resistor under the overcoated resin, and that the initial THI can be used to determine a specimen with a defective surface through a nondestructive test.

Next, the continuous variations of resistance are investigated for the group showing less than $-122 \mathrm{~dB}$ of THI.

\subsubsection{Small variation of resistance Figure 5 shows} the continuous variations of resistance under the high-temperature and humidity storage tests and the high-temperature storage test. Figure 6 also shows those under the electrical tests. The former shows that these changes are approximately linearly related to the square root of testing time. The latter shows complex variations of resistance because it was influenced by the self-heating and/or electric reaction under a bias voltage.

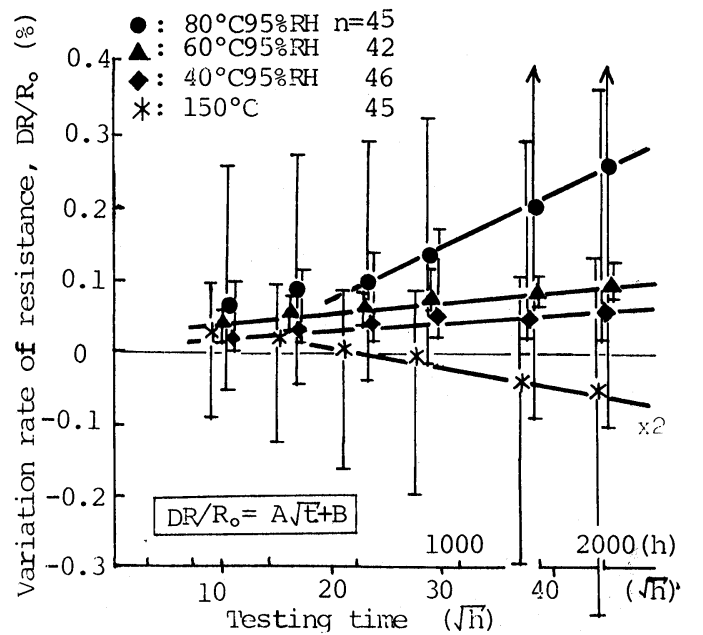

FIGURE 5 Continuous variation of resistance under various accelerated tests $(\mathrm{THI}<-122 \mathrm{~dB})$.

In addition, the resistance change of the common-conductor, $\mathrm{Au}-\mathrm{Pt}$ based thick film, was negligibly small. Therefore, the variation of resistance was caused by the variation of the ruthenium-based resistor.

\section{DISCUSSION}

In terms of experimental results, it becomes evident that the specimens of quite large THI showed a remarkable variation of resistance and almost all had a defective surface. 


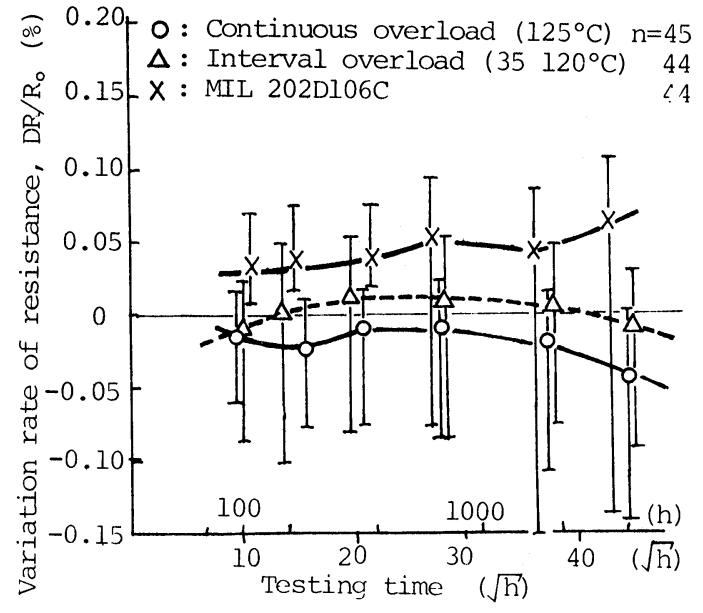

FIGURE 6 Continuous variation of resistance under various electrical tests (THI $<-122 \mathrm{~dB}$ ).

\subsection{Cause of a Variation of Resistance}

First, two experimental results are shown in order to substantiate the discussion of the cause of a variation of resistance.

1) Figure 7 shows the variation rate of resistance (DR/Ro) in the accelerated test under humidity and temperature stress. The resistance increased and decreased reversibly under humidity and temperature stress, respectively.

2) Figure 8 shows the Arrhenius plot of the DR/Ro during the high humidity test $(95 \% \mathrm{RH})$. These plots are approximately linear and show that the DR/Ro may be based upon an Arrhenius-type reaction. In these specimens, the activation energy is estimated at about $0.52 \mathrm{eV}$.

On the basis of the experimental results mentioned above, "the cause of a small variation of resistance" will be discussed.

1) The conduction component $\mathrm{RuO}_{2}$ reacts reversibly by hydration and dehydration, as indicated in the following chemical equation, causing the resistance to vary. ${ }^{4}$

$$
\mathrm{RuO}_{2}+\mathrm{xH}_{2} \mathrm{O} \leftrightarrow \mathrm{RuO}_{2} \cdot \mathrm{xH}_{2} \mathrm{O}
$$

2) The DR/Ro has a tendency to increase under high humidity conditions, and to decrease under high temperature conditions; i.e., dehydration conditions (Figure 5).

3) The DR/Ro increases and decreases reversibly under the influence of the cycling stress of humidity and temperature (Figure 7).

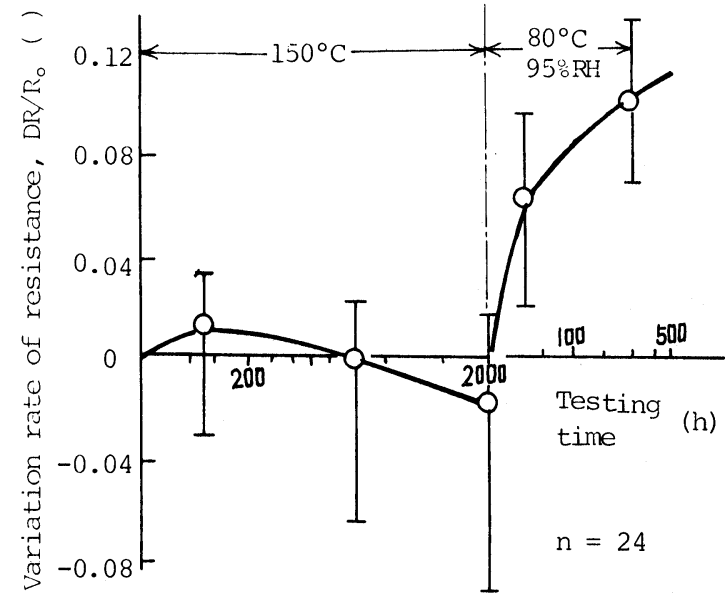

(a) High temperature and then high humidity storage test

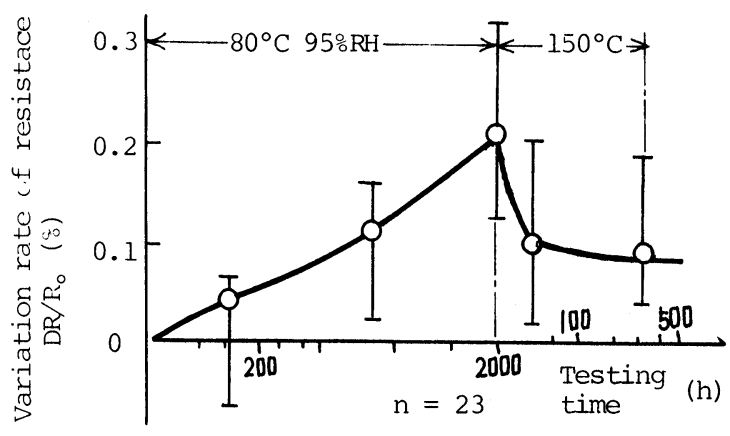

(b) High humidity and then high temperature storage test

FIGURE 7 Resistance change under the cycling stress of humidity and temperature.

4) The continuous variation of resistance is approximately linearly related to the square root of the testing time $t$ (Figure 5).

$$
\mathrm{DR} / \mathrm{Ro}=A \sqrt{t}+B
$$

where $A$ and $B$ are a constant.

5) The DR/Ro may be based upon an Arrhenius-type reaction (Figure 8).

6) The continuous variation of resistance of the $\mathrm{Au}-\mathrm{Pt}$ based common-conductor is negligibly small.

From all the above results, it seems that the small variation of resistance is mainly caused by hydration and dehydration in the conductive component $\mathrm{RuO}_{2}$ based upon "diffusion phenomenon" (for details of the consideration, see Appendix 1).

"Remarkable variations of resistance" may be caused by strong hydration and dehydration, because it 


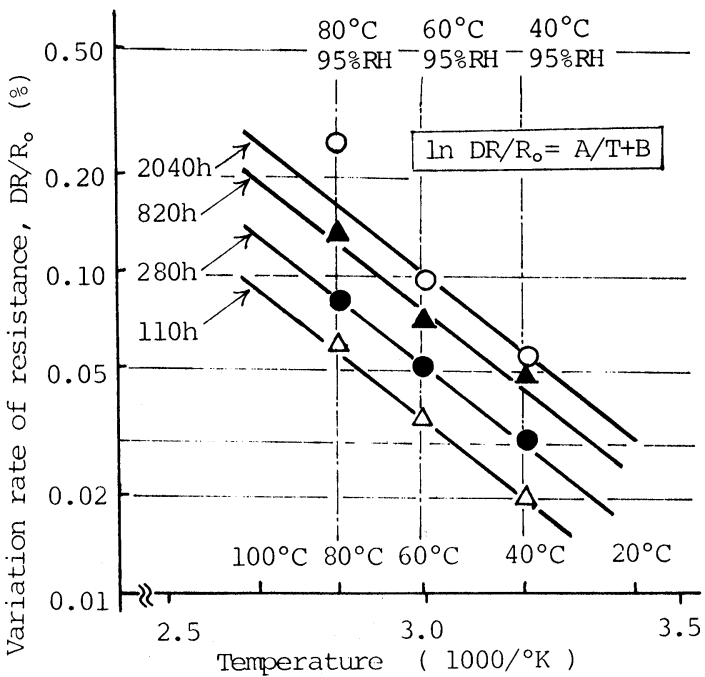

FIGURE 8 Arrhenius plot of the variation rate of resistance under high humidity storage test (95\% RH).

is easy for such chemical reactions to occur at uncovered parts of the overcoated glass where scratches and/or foreign substances exist. However, the effects of different conduction mechanisms in foreign substances, as well as shape changes and distortions of thick film resistors with scratches, are not negligible.

Next, the relationship between THI and DR/Ro is investigated to find the possibility of the reliability evaluation through measurement of THI.

\section{$4.2 T H I$ and $D R / R o$}

A relationship between the initial THI and the stability of a thin-film resistor are reported ${ }^{1,2,5}$ on the bases of experimental results. We explain the cause of different value of THI, considering from the structure of a thick film resistor.

4.2.1 Cause A small part of a conductive network within a thick-film resistor material is illustrated in Figure 9. Conductive paths are built-up by formation of three dimensional networks of ruthenate particles ${ }^{6}$ in the glass matrix during firing. Basically, there are two types of interconnected conductive particles of $\mathrm{RuO}_{2}$ : One is formed by the sintering of metal-oxide particles with each other, and the other is formed with thin interfacial regions between adjacent metal-oxide particles. The conductive mechanism ${ }^{7,8}$ through the insulated thin film in the latter type is of many kinds, as shown in Figure 9(c). An insulating barrier between particles can cause serious distortion. The sintering condition mentioned above depends upon many parameters, including the resistor material itself,

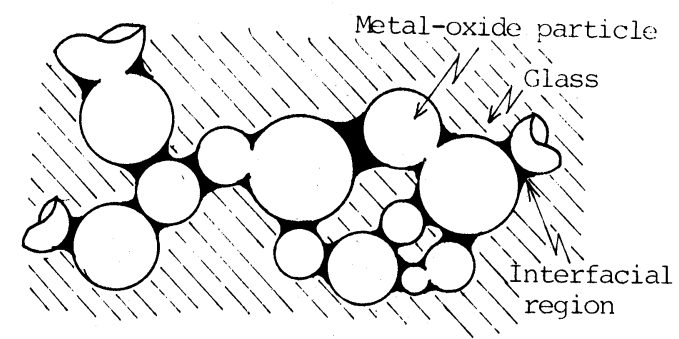

(a) An illustration of a conductive network 7 ) within a thick film resistor

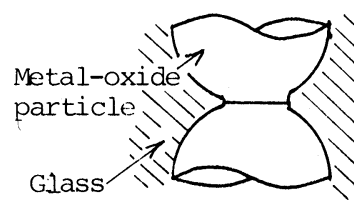

(b) Sintered contact

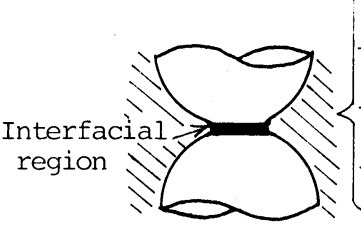

(c) Non-sintered contact

\section{Ohmic Conduction $\mathrm{J} \leadsto \mathrm{V} \exp (-\mathrm{C} / \mathrm{T})$}

Schottky Emission $\mathrm{J} \sim \mathrm{T}^{2} \exp [\mathrm{a} \sqrt{\mathrm{V}} / \mathrm{T}-\mathrm{q}(\Phi / \mathrm{kT})]$ Frenkel-Poole Emission $\sim V \cdot \exp [2 a \sqrt{V} / T-q(\phi / k T)]$ Tunnel or Field Emission $u V^{2} \exp (-\mathrm{b} / \mathrm{V})$ Space-Charge-Limited Ionic Conduction $\mathrm{V} / \mathrm{T} \cdot \exp (-\mathrm{d} / \mathrm{T})$
FIGURE 9 Schematic structure of thick film resistor.

processing conditions, admixtures of special components, etc. They may give rise to a nonlinearity and to the difference of THI.

The part with defects or impurities in these interfacial regions has the different value of a barrier of $\Phi$ volts to conduction electrons $q$ from surroundings. If an electric field $E$ is applied across this barrier $\Phi$, the current $I$ will give rise to odd harmonic components in the fluctuation of the current:

$$
\begin{aligned}
& I \propto N a v \exp (-\Phi / k T) 2 \sinh (q E a / 2 k T) \\
& \propto 2 N a v \exp (-\Phi / k t)\left[\frac{q V}{2 k T}+\frac{q^{3} V^{3}}{3 !(2 k t)^{3}}\right. \\
& \left.+\frac{q^{5} V^{5}}{5 !(2 k T)^{5}}+\cdots\right]
\end{aligned}
$$

$N$ : number of defects

$a$ : distance of lattice

$v$ : frequency of electron

$k$ : Boltzmann's constant

$T$ : temperature in ${ }^{\circ} \mathrm{K}$

$E a=V$ 
Neglecting at small currents the 5th and higher powers:

$$
I \simeq C_{1} V+C_{2} V^{3}
$$

where $C_{1}$ and $C_{2}$ are a constant.

Assume the pure sinusoidal loaded voltage:

$$
V=V_{0} \sin \omega t
$$

Trignometrically:

$$
\begin{aligned}
I & =C_{1} V_{0} \sin \omega t+C_{2} V_{0}^{3} \sin ^{3} \omega t \\
& =\left(C_{1} V_{0}+\frac{3}{4} C_{2} V_{0}^{3}\right) \sin \omega t-\frac{C_{2} V_{0}^{3}}{4} \sin 3 \omega t
\end{aligned}
$$

As a result, the 3 rd harmonic component is predominant. Therefore, the degree of resistor reliability can be estimated by THI corresponding to the difference of the structure of the thick film resistor itself.

\subsubsection{Relationship between THI and DR/Ro}

1) Figure 10 shows typical examples of the relationship between THI and DR/Ro at various testing times. Though DR/Ro varied in the accelerated test, the THI was almost constant.

2) DR/Ro may be mainly caused by hydration and dehydration in the conductive component $\mathrm{RuO}_{2}$.

Therefore, the factors which have an effect upon THI do not vary remarkably themselves, but their existence has an effect upon the amount and the reaction velocity of hydration and dehydration. For instance, due to

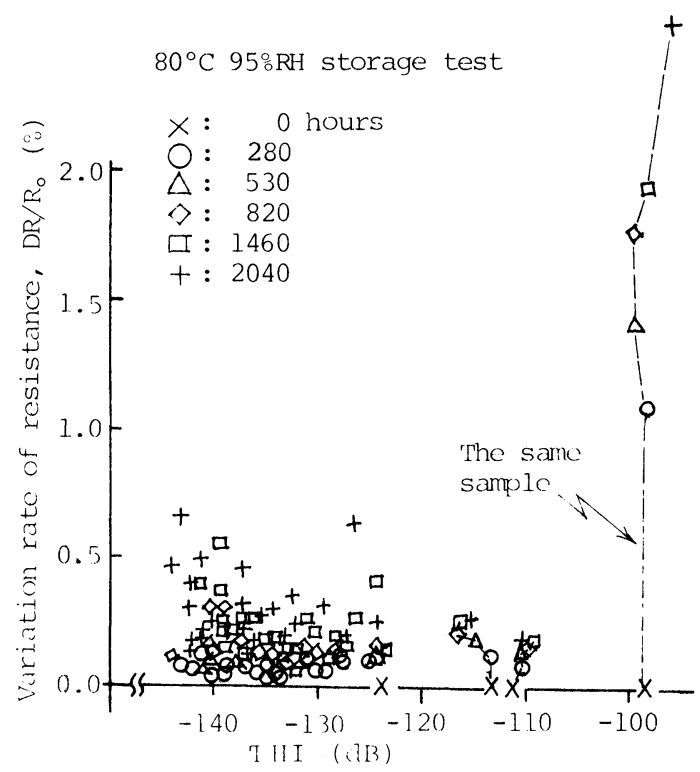

FIGURE 10 Relationship between Third Harmonic Index and variation rate of resistance. scratches and/or foreign substances on the surface of a resistor, there are large uncovered parts of overcoated glass where such a chemical reaction occurs easily.

As a result, it becomes evident that the initial THI (the degree of defect) is available to estimate the degree of resistor reliability.

\section{CONCLUSION}

A ruthenium-based thick film resistor was investigated in various accelerated tests, and the evaluation method of thick film resistors was studied by measuring the Third Harmonic Index (THI).

The results are concluded as follows:

1) It is found that there were two variation types of resistance in accelerate tests: One was quite large, while the other was small. The former was mainly due to scratches and/or foreign substances on the surface of a thick film resistor under the overcoated resin. The latter was caused by hydration and dehydration in conductive component $\mathrm{RuO}_{2}: \mathrm{RuO}_{2}+\mathrm{xH}_{2} \mathrm{O} \leftrightarrow$ $\mathrm{RuO}_{2} \cdot \mathrm{xH}_{2} \mathrm{O}$

2) A resistor which indicated quite large initial THI showed a remarkable variation of resistance, and had a defective surface in most cases. As a result, it becomes evident that the initial THI is available in order to estimate the degree of resistor reliability in a nondestructive test.

3) As the small variation of resistance is mainly caused by hydration and dehydration in the conductive component $\mathrm{RuO}_{2}$, on the basis of diffusion phenomenon, the evaluation of a lot is possible by the estimation of an activation energy by the Arrhenius plot of the variation rate of resistance under a temperature and humidity storage test.

\section{ACKNOWLEDGEMENTS}

The present authors would like to express their sincere thanks to Dr. S. Mitani, Chief Manager of our Reliability Center, for the useful guidance during the course of this study. Thanks go also to the persons in the factories concerned who kindly supplied specimens and information.

\section{REFERENCES}

1. A. Salomon and T. Troianello, Component Linearity Test improves Reliability Screening through Measurement of Third Harmonic Index, Annu. Proc. Reliab. Phys. Symp. 11th, 69-76 (1973).

2. J. C. Anderson and V. Rysanek, Prediction of the Stability of Thin-film Resistors, Radio and Electronic Eng. 39, 321-327 (June 1970). 
3. V. Peterson and Per-Olof Harris, Harmonic testing pinpoints passive component flaws, Electronics, 93-100, July 11 (1966).

4. Y. Takeda and M. Haradome, Stability and Deterioration Mechanism of Thick Film Resistors. Microelectronics and Reliability, 13, 281-289 (1974).

5. S. Hellstöm and H. Wesemeyer, Nonlinearity measurements of thin films, Vacuum 27 [4] 339-343 (1977).
6. E. H. Stevens et al., High-Voltage Damage and Low-Frequency Noise in Thick-Film Resistors, IEEE Trans. Parts Hybrids Packag. 12, 351-356 (1976).

7. R. W. Vest, Conduction mechanism in thick film microcircuits, Find Final Tech. Rep., Purdue Research Foundation (Dec. 1975).

8. G. E. Pike and C. H. Seager, Electrical properties and conduction mechanisms of Ru-based thick-film (cermet) resistors, J. Appl. Phys. 48, [12] 5152-5169 (Dec. 1977).

\section{Appendix 1}

\section{A CONSIDERATION FOR THE VARIATION OF RESISTANCE UNDER HIGH TEMPERATURE AND HUMIDITY STRESS}

For an example, considering the specimen under environmental conditions of constant relative humidity $\left(\mathrm{H}_{\mathrm{RH}}\right)$ and temperature $(\mathrm{T})$.

\section{Assumption 1}

A density of $\mathrm{H}_{2} \mathrm{O}$ vapour on the surface of a specimen is maintained at a constant value $\left(C_{0}\right)$.

Solving the equation of Fick's Law under the conditions of the Assumption 1, the total amount of $\mathrm{H}_{2} \mathrm{O}(Q)$ through the surface during the testing time $(t)$ is given by

$$
Q=2 C_{0} \sqrt{D_{0} / \pi} \cdot \sqrt{t} \cdot \exp \left(-E_{1} / 2 k T\right)
$$

$D_{0}$ : Diffusion constant

$E_{1}$ : Activation energy of the diffusion

$k$ : Boltzmann's constant

\section{Assumption 2}

The total amount of $\mathrm{H}_{2} \mathrm{O}(Q)$ reacts by the following chemical reaction and then the resistance changes.

$$
\begin{aligned}
& \mathrm{RuO}_{2}+\mathrm{xH}_{2} \mathrm{O} \leftrightarrow \mathrm{RuO}_{2} \cdot \mathrm{xH}_{2} \mathrm{O} \\
& \kappa=A \exp \left(-E_{2} / k T\right) \\
& \kappa: \text { Reaction velocity } \\
& E_{2}: \text { Activation energy of the reaction }
\end{aligned}
$$

\section{Assumption 3}

The variation rate of resistance $\left(D R / R_{0}\right)$ is in proportion to the total amount of $\mathrm{H}_{2} \mathrm{O}(Q)$.

$$
D R / R_{0} \propto Q
$$

\section{Assumption 4}

A density of $\mathrm{H}_{2} \mathrm{O}$ vapour $\left(C_{0}\right)$ is in proportion to the number of molecular of $\mathrm{H}_{2} \mathrm{O}$ vapour $(N)$ which collides against the surface of a specimen per unit time and unit area.

$$
\begin{aligned}
C_{0} \propto N & =B \cdot H_{R H} \cdot P_{s}(T) / \sqrt{2 \pi m k T} \\
& =B \cdot C \cdot H_{R H} \exp \left(-E_{3} / k T\right) / \sqrt{2 \pi m k T}
\end{aligned}
$$

$P_{S}(T)$ : Saturation vapour pressure

$E_{3}$ : Evaporation heat

From Eqs. (1)-(4), the variation rate of resistance under high temperature and humidity stress is given by

$$
D R / R_{0} \propto H_{R H} \cdot \sqrt{t} \cdot \frac{1}{\sqrt{T}} \cdot \exp \left\{-\left(E_{1} / 2+E_{2}+E_{3}\right) / k T\right\}
$$

In this case, the relative humidity $\left(H_{\mathrm{RH}}\right)$ is a constant and the variation rate of $1 / \sqrt{T}$ is negligibly small compared with the one of $\exp \left\{-\left(E_{1} / 2+E_{2}+E_{3}\right) / k T\right\}$ when temperature $(T)$ varies.

\section{Therefore}

$\begin{array}{ll}D R / R_{0}=C_{1} \sqrt{t}+C_{2} & \text { Figure } 5(T \text { is a constant }) \\ \ln \left(D R / R_{0}\right)=-C_{3} / T+C_{4} & \text { Figure } 8(t \text { is a constant })\end{array}$ where $C_{1}, C_{2}, C_{3}$ and $C_{4}$ are a constant. 

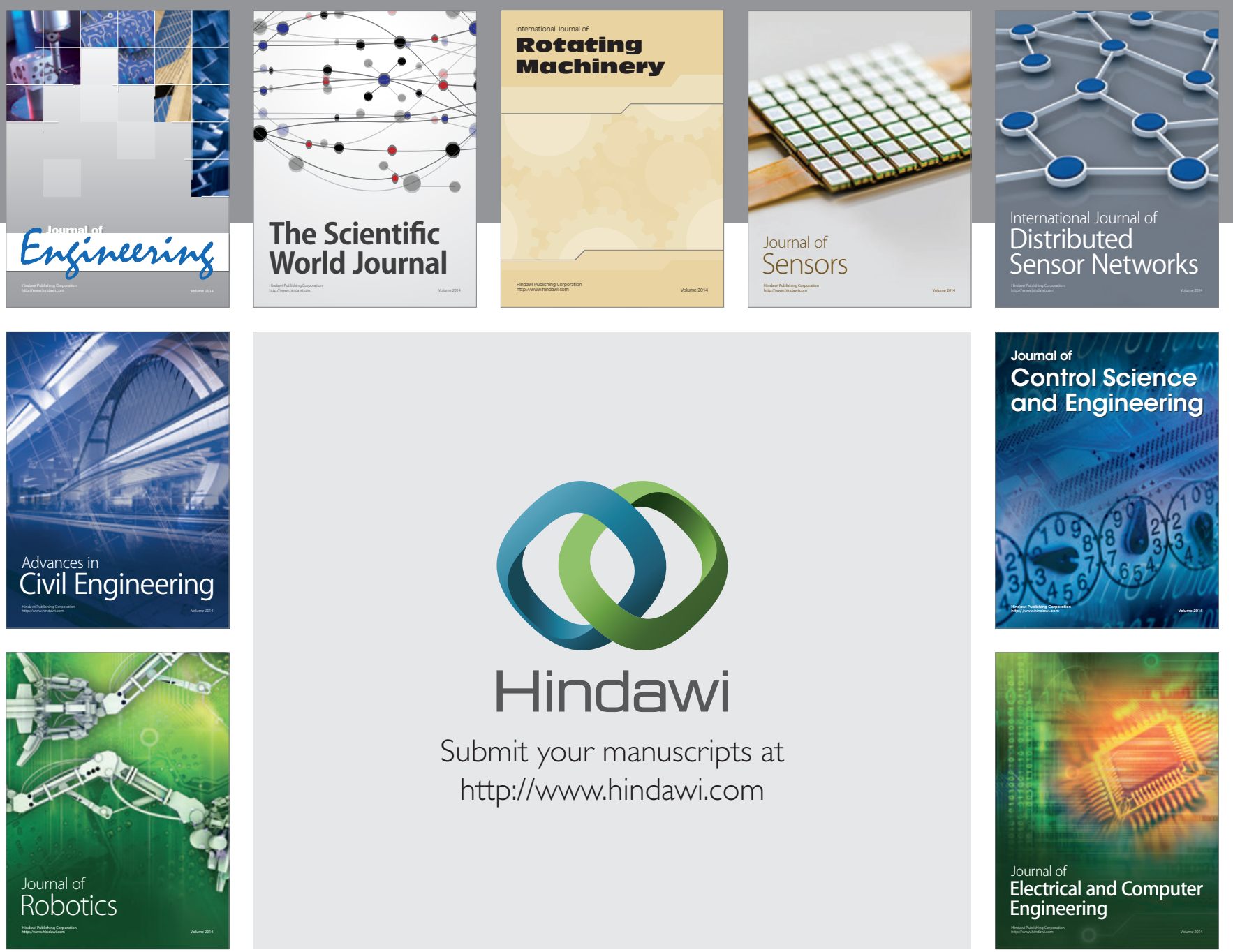

Submit your manuscripts at

http://www.hindawi.com
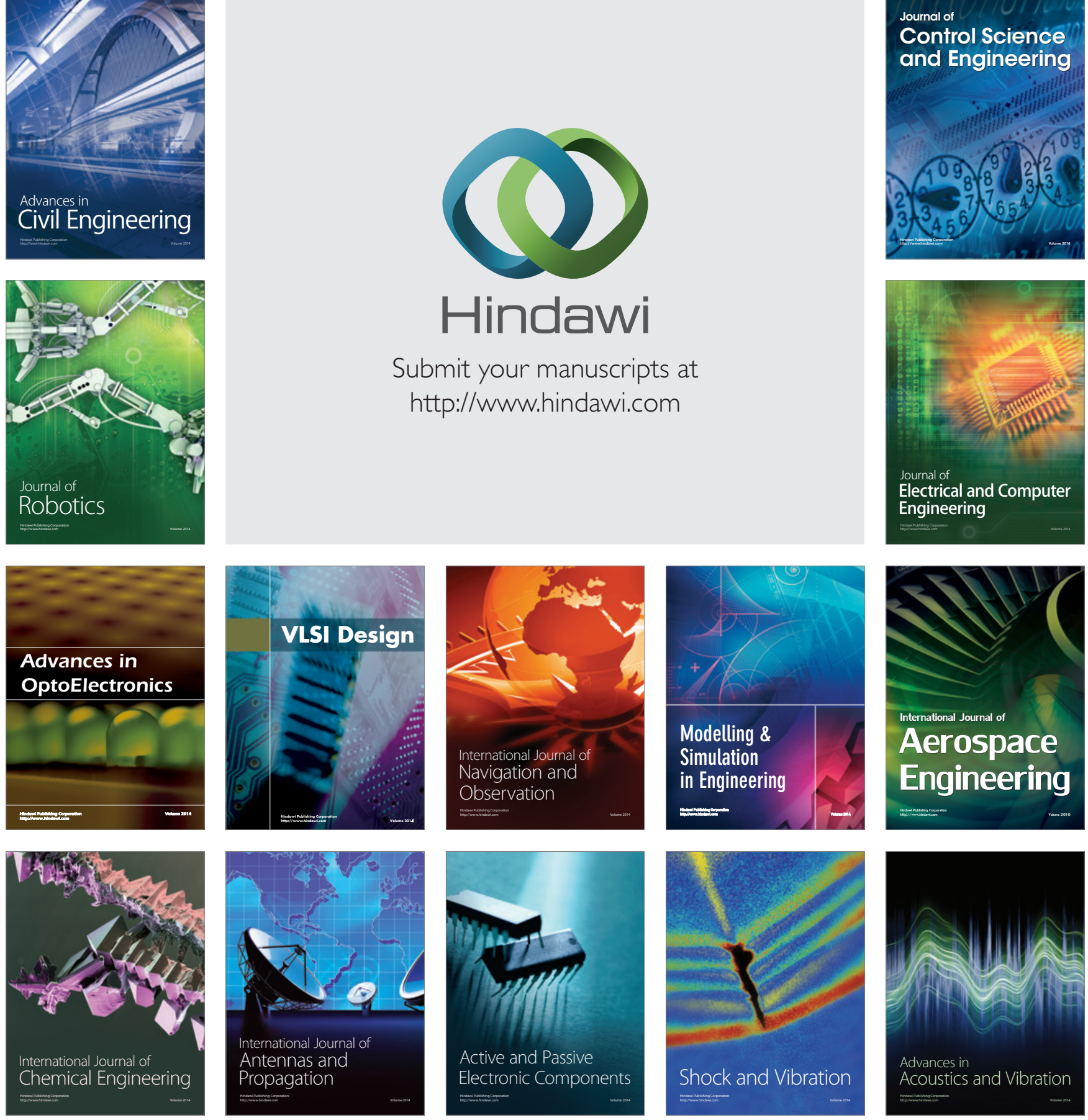